\title{
Use of Volume Measurement in Adrenal Mass Follow Up
}

\author{
Arzu Turan (Corresponding author) \\ Recep Tayyip Erdogan University, Medicine Faculty, Department Of Radiology, Rize, Turkey \\ ORCID ID:0000000163988202 \\ E-mail: rztrn72@gmail.com \\ Fatma Beyazal Celiker \\ Recep Tayyip Erdogan University, Medicine Faculty, Department Of Radiology, Rize, Turkey \\ ORCID ID:0000000254209825 \\ E-mail:fabece@yahoo.com \\ Eda Beykoz Cetin \\ Recep Tayyip Erdogan University, Medicine Faculty, Department Of Radiology, Rize, Turkey \\ E-mail: edabeykozcetin@ hotmail.com \\ Hatice Beyazal Polat \\ Recep Tayyip Erdoğan University, MedicineFaculty, Department Of Internal Medicine, Rize, Turkey \\ E-mail: drpolat53@gmail.com
}

\begin{abstract}
Purpose:

The purpose of our study was to assess the use of volume measurement in addition to or together with linear measurement in adrenal mass follow up. Since the morphologies of the adrenal gland may differ (such as Y,Z, andV), adrenal masses may also be visualized in different geometrical forms.If adrenal masses are hormonally inactive and do not exhibit significant changes in dimension they can be follow up for years, and measurements can therefore exhibitinterobserver differences.
\end{abstract}

Method:

Linear measurements and manual and automatic volume values obtained from first abdominal computerized tomography (CT) images from 49 patients under monitoring for adrenal adenoma were compared with final control abdominal CT measurements. No significant difference was observed in any measurements. We observed $99.7 \%$ correlation between 'first linear measurement' and' first volume,' and a similar level between 'final linear measurement' and 'final volume.'

Conclusion:

Volume measurement can be performed separately or together with three-dimensionallinear measurements in adrenal mass follow up.

DOI: $10.7176 / \mathrm{JSTR} / 5-2-16$

\section{INTRODUCTION}

The adrenal gland may exhibit different morphological variants. Similarly, adrenal masses may exhibit different geometrical patterns $\left({ }^{1}\right)$. The risk of malignancy in adrenal adenomas in quite low, $\left({ }^{2}\right)$ and followup may continue for up to 5 years. If adrenal adenomas are hormonally inactive and do not exhibit significant changes in dimension $(\geq 5 \mathrm{~mm})$ they can be monitored for a number of years, and measurements can therefore exhibit interobserver differences $\left({ }^{2}\right.$. The ability of computerized tomography (CT) to show glandular extension is diagnostically significant, and the high accuracy of adrenal volume measurement at CT means that volume measurement with CT is highly practicable $\left(3^{3 / 4}\right)$. In our study, axial and reconstructed coronal plane images, on which the adrenal mass is best visualized, were obtained from first and final control abdominal CT images from 49 patients with hormonally inactive adrenal adenomas ${ }^{6}$. Data were analyzed with three-dimensional linear measurements and volume measurements taken from these sections. The guidelines recommend surgical resection in the event of $20 \%$ or higher growth of the adrenal mass (in addition to an increase of at least $5 \mathrm{~mm}$ in maximum diameter) ${ }^{7}$. An increase of $\geq 5 \mathrm{~mm}$ 
in three-dimensional linear measurement and a volume increase exceeding $20 \%$ were observed in 11 patients $(22.5 \%)$ in our study, but resection was not recommended since these were hormonally inactive at follow-up.

Volume measurement is employed in incidentaloma follow-up, monitoring at extended intervals will facilitate comparative assessment. Similarly to prostate monitoring, we think that three-dimensional linear measurement $(\mathrm{mm})$ and volume $\left(\mathrm{mm}^{3}\right)$ can be used together with volume measurement $\left(\mathrm{mm}^{3}\right)$ to monitor adrenal masses. The addition of volume to these measurements will contribute to the effectiveness of follow-up.

\section{Materials and Methods \\ Ethical Approval}

Approval for the study was granted by our university ethical committee (No. 40465587-62 dated 06.04.2017) and the training research hospital administration.

\section{Patients}

Abdominal CT images from 49 patients followed-up due to adrenal mass between 2014 and 2017 were evaluated retrospectively. Theseconsisted of patients referred to the radiology unit for abdominal CT under the adrenal mass follow-up protocol. Patients with preliminary diagnosis ofadrenal adenoma and undergoing at least two consecutive abdominal CT sessions were included in the study. Patients with endocrine disease, septic shock, trauma, depression, adrenalectomy, and long-term therapy with glucocorticoids were excluded. Medical information recorded on the hospital system was investigated. Biochemical and endocrine analyses(metanephrine, cortisol, dexamethasone suppression test, aldosterone, renin, HbA1c, DHEA-sulfate, ACTH, 25OH Vitamin D, and insulin values), endoscopy results if available (for investigating the presence of accompanying gastritis), type of operation and pathology data were examined. Levels of metanephrine, normetanephrine, and vanillylmandelic acid in 24-h urine were examined at pre-operative endocrinological examination of all patients recommended for surgical resection. In hypertensive cases, plasma aldosterone concentration, plasma renin activity and the aldosterone/renin ratio were also analyzed following adjustment of antihypertensive therapy such as not to affect the renin-angiotensin-aldosterone system in order to investigate primary hyperaldosteronism. The analysis results were evaluated, and patients with a preliminary diagnosis of hormonally inactive adrenal adenoma were included in the study.

\section{CT scan protocol}

All abdominal CT imaging was performed with a 256slice-6 mm CT scanner (Somatom Sensation 256, Siemens Medical Solutions, Germany). Rotation time was $0.33 \mathrm{sec}$, reference tube current $220 \mathrm{~mA}$ and tube voltage $120 \mathrm{kVp}$. The thickness and reconstruction range in the images obtained were standardized at $0.625 \mathrm{~mm}$. Window width and level for volume and linear measurements were at standard abdominal settings.

For contrast increase, $1 \mathrm{ml} / \mathrm{kg}$ contrast materialwas injected into an antecubital vein with a $3 \mathrm{~mL} / \mathrm{sec}$ flow rate using an18-21 catheter. Automatic determination of the peak increase in theabdominal aorta was used for bolus timing. Three-phase (arterial-venous-late $(15 \mathrm{~min})$ ) abdominal CT scanning was performed.

\section{Measurements}

Axial and coronal sections on which the adrenal mass was best visualized were obtained from initial and final control abdominal CT images from 49 patients under follow-up for hormonally inactive adrenal adenoma as outlined below:

First, linear measurements of the mass were performed in $\mathrm{mm}$ in three planes -inferosuperior (height), ventrodorsal (depth) and mediolateral (width from reconstructed coronal images).

Second, manual volume estimations of the masses were performed from three-dimensional linear measurements using the formula height $(\mathrm{mm}) \mathrm{x}$ depth $(\mathrm{mm}) \mathrm{x}$ width $(\mathrm{mm}) \mathrm{x} 0.523 / 0.5(\mathrm{~cm} 3)$.

Third, estimated $20 \%$ growth amounts were calculated for each mass using manual volume values with the formula(initial volume $\mathrm{x} 20$ )/ 100+(initial volume).

Fourth, automatic volumes were calculated using commercially available software (AW-server, GE, andsyngo Volume, Siemens) in 25 patients capable of being transported to the workstation from adrenal masses subjected to manual volume measurement in order to assess any potential difference between automatic or manual calculation in mass volume measurements (Figure 1). 


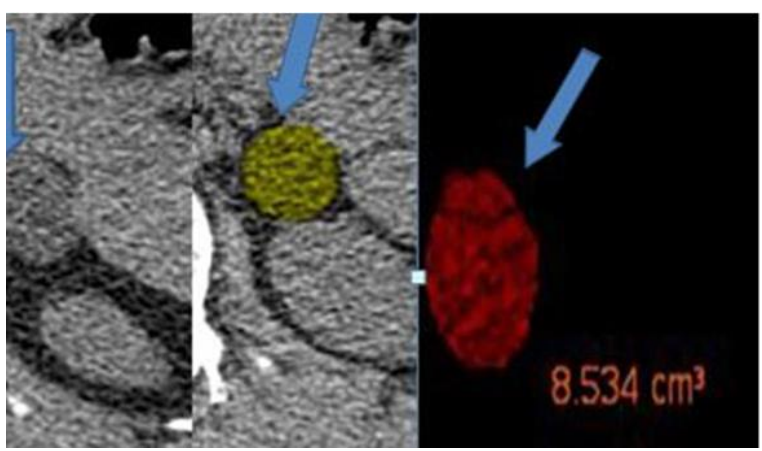

Figure 1. Automatic calculation in mass volume measurements

Fifth, linear measurement and three-dimensional volume values obtained from first and final control abdominal CTs were subjected to statistical analysis.

\section{Statistical analysis}

Descriptive statistics for continuous (numerical) variables in this study of volume measurement for use in adrenal adenoma were expressed as mean, standard deviation, minimum andmaximum values, while categorical variables were expressed as number $(\mathrm{N})$ and percentage $(\%)$.

The Shapiro-Wilk test was applied to determine whether the volume values obtained were compatible with normal distribution $(\mathrm{N}<50)$ (Table 1). All volume measurements were determined not to be normally distributed according to both tests $(\mathrm{p}<0.05)$. Non-parametric tests were therefore applied. All calculations were performed onSPSS (IBM SPSS for Windows, Ver.24) software.

\section{Results}

The patients consisted of 23 men (46.9\%) and 26 women (53.1\%) with a mean age of59 \pm 9 years. Followup durations were one year in two patients $(4.1 \%)$, two years in $15(30.6 \%)$, three years in $17(34.7 \%)$, four years in six(12.2\%), and five years in nine(18.4\%). Endoscopy was performed on 12 of these patients, and various stages of gastritis (active, chronic, or superficial) were determined in all (Table2).

General descriptive statistics for volume and three-dimensional linear measurements are shown in Table 3. Mean values were determined, and the distribution of all measurements was shown in graph form. Volume measurements and three-dimensional linear measurements were then compared (Table 4). 'Automatic volume' produced values close to those of 'manual volume obtained from three-dimensional linear measurement.'Finally, the statistical significance of differences between the volume measurements was analyzed (Table5). Accordingly, a significant difference was determined between first and final volumes $(p=0.011)$. Similarly, a significant difference was determined between first volume and threedimensional linear measurement $(\mathrm{p}<0.001)$.

In summary, all two-way comparisons yielded statistically significant differences $(\mathrm{p}<0.05)$, and results for three-dimensional linear measurements were more effective than those elicited from first and final volume. Correlation analysis of manual and automatic volume measurements revealed a high level of correlation between all measurements, with $99.7 \%$ correlation between first three-dimensional linear measurement and first volume. The correlation between these measurements is also shown as a scatter plot.

Table 1. All volume measurements were normally distributed at volume and linear measurement analysis using the Shapiro-Wilk $(\mathrm{N}<50)$ test $(\mathrm{p}<0.05)$

\begin{tabular}{|lc|c|c|c|c|c|}
\hline & \multicolumn{2}{c}{ Kolmogorov-Smirnov } & \multicolumn{3}{c|}{ Shapiro-Wilk } \\
& Statistic & df & p. & Statistic & df & p. \\
\hline First volume &, 212 & 23 &, 009 &, 799 & 23 & $<, 001$ \\
\hline Final control volume &, 271 & 23 & $<, 001$ &, 770 & 23 & $<, 001$ \\
\hline First linear measurement &, 209 & 23 &, 010 &, 807 & 23 & $<, 001$ \\
\hline Final linear measurement &, 241 & 23 &, 001 &, 799 & 23 & $<, 001$ \\
\hline Auto-volume &, 239 & 23 &, 001 &, 803 & 23 & $<, 001$ \\
\hline
\end{tabular}

$d f:$ Degree offreedom 
Table 2. Patients' demographic and laboratory data

\begin{tabular}{|c|c|}
\hline Male & $(46,9 \%)$ \\
\hline Female & $(53,1 \%)$ \\
\hline \multirow{2}{*}{ Age (mean) } & $59 \pm 9$ \\
\hline & Range \\
\hline metanephrin $\quad$ (mcg/day) & $50-250$ \\
\hline Dexamethasonesuppression test & $1<$ \\
\hline cortisol $\quad(\mathrm{ug} / \mathrm{dL})$ & $3,7-19,4$ \\
\hline Aldosteron $\quad$ (ng/ml/hour) & \\
\hline $\begin{array}{ll}\text { Renin } & \text { (ng/ml/hour) } \\
\end{array}$ & \\
\hline HbA1c $\quad(\mathrm{mmol} / \mathrm{ml})$ & $20-42$ \\
\hline DHEA- sulfate $\quad(\mu \mathrm{g} / \mathrm{dL})$ & $35-430$ \\
\hline ACTH $\quad(\mathrm{pg} / \mathrm{mL})$ & $0-46$ \\
\hline 25-OH Vitamin D $\quad(\mathrm{ng} / \mathrm{ml})$ & $9,5-55,5$ \\
\hline $\begin{array}{ll}\text { İnsülin } \quad(\mu \mathrm{U} / \mathrm{mL}) \\
\end{array}$ & $5-10$ \\
\hline Endoscopyresults & active, chronicgastritis (n: 12) \\
\hline \multicolumn{2}{|l|}{ surgicaloperation } \\
\hline Pathology & \\
\hline
\end{tabular}

Table 3. General descriptive statistics for volume and linear measurements; first volume and final control volumes were low, and three plane linear measurement values were higher than other measurements.

However, automatic volume produced a result close to the three plane linear measurement values

\begin{tabular}{|l|r|r|r|r|r|r|}
\hline \multicolumn{1}{|c|}{ N } & Median & \multicolumn{1}{c|}{ Mean } & Std. Dev. & Min. & \multicolumn{1}{c|}{ Max. } \\
\hline First volume & 49 & 3,91 & 6,0302 & 5,92642 &, 36 & 26,20 \\
\hline Final controlvolume & 47 & 4,14 & 7,8006 & 9,22048 &, 30 & 44,29 \\
\hline First linearmeasurement & 49 & 7,750 & 11,72612 & 11,642752 &, 580 & 52,320 \\
\hline Final linearmeasurement & 48 & 8,140 & 15,46479 & 17,796315 &, 590 & 84,500 \\
\hline Auto-volume & 24 & 7,56 & 10,5720 & 11,85912 &, 26 & 45,70 \\
\hline
\end{tabular}

Table 4. Statistical comparisons of differences between volume and linear measurements; a significant difference was determined between first volume and final volume (pair 1) ( $\mathrm{p}=0.011$ ). Similarly, a significant difference was determined between first volume and three-dimensional linear measurement (pair 2) $(\mathrm{p}<0.001)$. In other words, three-dimensional linear measurement was more effective. A significant change was also determined between first volume and final linear measurement $(p<0.05)$. We concluded that final linear measurements are more effective than first volume $(\mathrm{p}<0.05)$.

\begin{tabular}{|c|c|c|c|c|c|c|}
\hline & & $\mathbf{N}$ & Mean & Difference & Std. Dev. & $* \mathbf{p}$. \\
\hline \multirow{2}{*}{ Pair 1} & First volume & 47 & 5,8580 & \multirow{2}{*}{$-1,9426$} & 5,95328 & \multirow[t]{2}{*}{,011 } \\
\hline & Final control volume & 47 & 7,8006 & & 9,22048 & \\
\hline \multirow{2}{*}{ Pair 2} & First volume & 49 & 6,0302 & \multirow{2}{*}{$-5,69596$} & 5,92642 & \multirow[t]{2}{*}{$<, 001$} \\
\hline & First linear measurement & 49 & 11,73 & & 11,643 & \\
\hline \multirow{2}{*}{ Pair 3} & First volume & 48 & 5,8777 & \multirow{2}{*}{$-9,58713$} & 5,89118 & \multirow[t]{2}{*}{$<, 001$} \\
\hline & Final linear measurement & 48 & 15,46 & & 17,796 & \\
\hline \multirow{2}{*}{ Pair 4} & First volume & 24 & 6,5095 & \multirow{2}{*}{$-4,06246$} & 6,70455 & \multirow[t]{2}{*}{,005 } \\
\hline & Auto-volume & 24 & 10,5720 & & 11,85912 & \\
\hline \multirow{2}{*}{ Pair 5} & Final control volume & 47 & 7,8006 & \multirow{2}{*}{$-3,59149$} & 9,22048 & \multirow[t]{2}{*}{$<, 001$} \\
\hline & First linear measurement & 47 & 11,39 & & 11,709 & \\
\hline \multirow{2}{*}{ Pair 6} & Final control volume & 47 & 7,8006 & \multirow{2}{*}{$-7,64851$} & 9,22048 & \multirow[t]{2}{*}{$<, 001$} \\
\hline & Final linear measurement & 47 & 15,45 & & 17,988 & \\
\hline \multirow{2}{*}{ Pair 7} & Final controlvolume & 23 & 7,9904 & \multirow{2}{*}{$-2,68465$} & 9,22087 & \multirow[t]{2}{*}{100} \\
\hline & Auto-volume & 23 & 10,6751 & & 12,11464 & \\
\hline \multirow{2}{*}{ Pair 8} & First linear measurement & 48 & 11,44 & \multirow{2}{*}{$-4,026$} & 11,588 & \multirow[t]{2}{*}{, 003} \\
\hline & Final linear measurement & 48 & 15,46 & & 17,796 & \\
\hline \multirow{2}{*}{ Pair 9} & First linear measurement & 24 & 12,88 & \multirow{2}{*}{2,30638} & 13,344 & \multirow[t]{2}{*}{, 014} \\
\hline & Auto-volume & 24 & 10,5720 & & 11,85912 & \\
\hline \multirow{2}{*}{ Pair 10} & Final linear measurement & 24 & 16,49 & \multirow{2}{*}{5,91304} & 17,649 & \multirow[t]{2}{*}{,002 } \\
\hline & Auto-volume & 24 & 10,5720 & & 11,85912 & \\
\hline
\end{tabular}

137 | P a g e

www.iiste.org 
Table 5. Correlation coefficients between measurements; all measurements were compared with one another. Statistically significant correlations are indicated by asterisks $(*)(\mathrm{p}<0.01)$. High correlation was determined between all measurements. For example, 99.7\% correlation was determined between first linear measurement and first volume.

\begin{tabular}{|lcc|c|c|c|}
\hline & & $\begin{array}{c}\text { First } \\
\text { volume }\end{array}$ & $\begin{array}{c}\text { Final control } \\
\text { volume }\end{array}$ & $\begin{array}{c}\text { First linear } \\
\text { measurement }\end{array}$ & $\begin{array}{c}\text { Final linear } \\
\text { measurement }\end{array}$ \\
\hline Final control volume & $\mathrm{R}$ &, $918^{* *}$ & & & \\
\hline $\begin{array}{l}\text { First linear } \\
\text { measurement }\end{array}$ & $\mathrm{R}$ &, $997^{* *}$ &, $916^{* *}$ & & \\
\hline $\begin{array}{l}\text { Final linear } \\
\text { measurement }\end{array}$ & $\mathrm{R}$ &, $922^{* *}$ &, $950^{* *}$ &, $925^{* *}$ & \\
\hline Auto-volume & $\mathrm{R}$ &, $746^{* *}$ &, $838^{* *}$ &, $750^{* *}$ &, $769^{* *}$ \\
\hline
\end{tabular}

$R:$ Spearman's correlation coefficients

$* * P<0.01$

\section{Discussion}

This study evaluated the use of volume measurement in the monitoring of adrenal masses. Abdominal CT is the most widely employed sectional imaging method for evaluating the adrenal glands $\left({ }^{1{ }^{1} 8}\right)$, particularly due to its ability to show glandular extension. A high level of accuracy in volume determination with CT makes adrenal volume measurement with CT particularly practicable $\left(^{3 / 4}\right)$.

The recommendations for the management of adrenal incidentaloma in clinical guidelines may be listed as follows: $\left(^{7}\right)$ When an adrenal mass is first detected, the clinician should aim to determine whether it is benign or malignant. The application of an imaging procedure, non-contrast CT scanning, is recommended in alladrenal incidentalomas to determine whether the mass is homogeneous and rich in lipids $\left({ }^{7 / 2}\right)$. These guidelines seek to answer four important questions during the monitoring of adrenal masses; how the risk of malignancy will be assessed with imaging, how Cushing's syndrome will be identified and managed, which patients should receive surgical treatment, and what are the monitoring indications if the adrenal mass cannot be excised surgically? $\left({ }^{2}\right)$. The recommendations include patients with adrenal masses not considered for surgical resection following initial evaluation but undergoing growth re-assessment with non-contrast CT or MRI after 6-12 months $\left({ }^{7}\right)$. In addition, the guidelines recommend surgical resection of the adrenal mass in case of growth of $20 \%$ or more (in addition to an increase in diameter of at least $5 \mathrm{~mm}$ ) during follow-up $\left({ }^{2}\right)$, while long-term monitoring is restricted to lesions of $4 \mathrm{~cm}$ and less (612 months) and an increase in volume less than $20 \%\left({ }^{7}\right)$. This will result in a safe approach being maintained in the management of adrenal masses. While there have been other studies of adrenal gland volume measurement, we encountered no previous studies of the use of volume in adrenal adenoma follow-up.

Our hospital's endocrinology polyclinic adrenal mass monitoring protocol recommends biochemical and radiological follow-up if the mass is $4 \mathrm{~cm}$ in size or less, monitoring at a range of $4-6 \mathrm{~cm}$ if the mass is hormonally inactive and radiological imaging suggests that it is benign, and surgical resection if it is larger than $6 \mathrm{~cm}$ or hormonally active $\left({ }^{9}\right)$. In our study we observed an increase of $5 \mathrm{~mm}$ or more at linear measurement and a volume increase exceeding $20 \%$ in 11 of the 49 cases $(22.5 \%)$, but resection was not advised since these were hormonally inactive.

One critical question not addressed in the guideline concerns biochemical analysis when an adrenal incidentaloma is first detected and the radiologist's subsequent monitoring protocol. The literature recommends that all adrenal incidentalomas be subjected to endocrinological and biochemical evaluation $\left({ }^{7}\right)$. Interobserver observation was present in those of our cases with increases of $5 \mathrm{~mm}$ or more at three-dimensional linear measurements $\left({ }^{10}\right)$. We think that when volume measurement is employed in incidentaloma follow-up, monitoring at extended intervals will facilitate comparative assessment. Similarly to prostate monitoring, we think that three-dimensional linear measurement (height $(\mathrm{mm}) \mathrm{x}$ depth $(\mathrm{mm}) \mathrm{x}$ width $(\mathrm{mm})$ and volume $\left(\mathrm{mm}^{3}\right)$ ) can be used together with volume measurement $\left(\mathrm{mm}^{3}\right)$ to monitor adrenal masses. Further prospective controlled studies involving real postoperative volume correlation with larger sample groups are now needed. 


\section{Limitations}

1. The fact that this is the first study of volume measurement in adenoma follow-up,

2. The volume values obtained could not be confirmed with true volumes,

3. The effects of sex, weight and BMI on volume measurements were not evaluated, and

4. The retrospective nature of the study.

\section{References}

1. J. M. Vincent, I. D. Morrison, P. Armstrong RHR. The Size of Normal Adrenal Glands on Computed Tomography. Clin Radiol. 1994;49:453-455.

2. Society E, Network E, Tumors A, Society FE. European recommendations for the management of adrenal incidentalomas : A debate on patients follow-up. Ann d'Endocrinologie. 2017;(2):8-11.

3. Schneller J, Reiser M, Beuschlein F, et al. Linear and Volumetric Evaluation of the Adrenal Gland - MDCT-Based Measurements of the Adrenals. Acad Radiol. 2014;21(11):1465-1474.

4. Benchekroun G, Garnier F, Delisle F, Rosenberg D. Use of Computed Tomography Adrenal Gland Measurement for Differentiating ACTH Dependence from ACTH Independence in 64 Dogs with Hyperadenocorticism. J Vet Intern Med. 2011;25:1066-1074.

5. Nougaret S, Jung B, Aufort S, Chanques G, Jaber S, Gallix B. Adrenal gland volume measurement in septic shock and control patients: A pilot study. Eur Radiol. 2010;20(10):2348-2357.

6. Carsin-vu A, Oubaya N, Mulé S, et al. Urogenital MDCT Linear and Volumetric Analysis of Adrenal Glands: Normative Data and Multiparametric Assessment. Eur Radiol 2016 Aug;26(8)2494-501.

7. Sahdev A. Recommendations for the management of adrenal incidentalomas : what is pertinent for radiologists ? Br J Radiol. 2017;(July 2016):90:20160627.

8. Wang X, Jin Z, Xue H, et al. Evaluation of Normal Adrenal Gland Volume by 64-slice CT. Chinese Med Sci J. 2012;27(4):220-224. http://dx.doi.org/10.1016/S1001-9294(13)60005-X.

9. Fassnacht M, Arlt W, Bancos I, Dralle H. Management of adrenal incidentalomas : European Society of Endocrinology Clinical Practice Guideline in collaboration with the European Network for the Study of Adrenal Tumors. Eur J Endocrinol. 2016;(175):G1-G34.

10. Mosconi C, Vicennati V, Dalmazi G Di, et al. Can Imaging Predict Subclinical Cortisol Secretion in Patients With Adrenal Adenomas? A CT Predictive Score. AJR. 2017;(July):1-8. 